\title{
Immune recognition of OxLDL in atherosclerosis
}

\author{
John F. Kearney
}

Department of Microbiology, Division of Developmental and Clinical Immunology, University of Alabama at Birmingham, Birmingham, Alabama 35294, USA.

Phone: (205) 934-6557; Fax: (205) 934-1875; E-mail: john.kearney@ccc.uab.edu.

The innate nonadaptive immune system plays a crucial role in the acute response to infectious agents. Within hours of exposure to an invading microorganism, well before adaptive cellular or humoral immune responses are engaged, the bulk of most infections is cleared by cells and molecules that bind with broad specificity to structures common to various pathogens. Only when infectious organisms break through these barriers do antigen receptor-bearing effector cells of the adaptive immune system come into play, ensuring that these organisms are cleared.

Blurring the distinction between the innate and the cognate aspects of the immune system are subsets of lymphocytes that recognize pathogenic determinants and are expressed even in immunologically naive animals (1). The significance of these lymphocytes is shown by the strong protection, elicited by $\mathrm{T}$-independent natural IgM antibody, against certain viral and bacterial infections (2). The B- or T-cell receptors of these lymphocytes often contain highly conserved sequences, arise reproducibly in all mice, and do not depend on exogenous antigen for their generation. In this respect, they constitute an "evolutionary memory" and form a bridge between pattern recognition molecules - mannose receptors, macrophage scavenger receptors, and other similar molecules (3) and the classic adaptive responses, which generally require T-cell help both to hone the specificity of the molecular interaction and to maintain immunological memory. These evolutionarily conserved responses to ubiquitously expressed microbial components are not confined to B cells, as there are numerous examples of $\alpha \beta$ and $\gamma \delta$ T cells that express canonical receptors to microbial components $(4,5)$.
In this issue of the JCI, Shaw et al. (6) show that these structurally distinct receptors functioning in different branches of the immune system nonetheless share patterns of ligand specificity. This provocative finding not only suggests mechanisms that may influence the progression of the disease, but also identifies a hitherto unknown function of an antibody response to a pathogenic bacterium.

\section{Natural antibodies to phosphorylcholine}

One manifestation of the chronic inflammatory process in $A p o E^{-/-}$mice, which provide a commonly studied animal model for atherosclerosis, is the vigorous induction of autoantibodies to oxidized LDL (OxLDL). Working with this disease model, Shaw et al. (6) also bridges patterns in microbial components.

show that these antibodies belong to a class of highly conserved natural antibodies with the so-called T15 idiotype. These antibodies are known to participate in the immune response to phosphorylcholine (PC) and to confer protection from organisms such as Streptococcus pneumoniae.

PC is a component of multiple prokaryotic and eukaryotic organisms. In S. pneumoniae, it is associated with the cell wall component teichoic acid via $\mathrm{N}$-acetylgalactosamine, and in a variety of other Gram-positive organisms, including other Streptococcus, Clostridium, and Bacillus species, PC is associated with different sugar residues. This lipid is also found in a variety of disease-inducing protozoa, fungi, nematodes, and other intestinal parasites (1). The immune response to $\mathrm{PC}$ in mice and its role in inducing protective antibodies against virulent $S$. pneumoniae infection are well described. When administered in the form of a heatkilled $S$. pneumoniae vaccine, the antibody response to $\mathrm{PC}$ is largely $\mathrm{T}$-independent (IgM and IgG3) and oligoclonal, being dominated by antibodies containing a canonical $\mathrm{V}_{\mathrm{H}} \mathrm{DJ}_{\mathrm{H}^{-}}$ $\mathrm{V}_{\mathrm{K}} 22 \mathrm{~J}_{\mathrm{K}} 5 \mathrm{H}+\mathrm{L} \mathrm{Ig}$ chain combination.

This combination of $\mathrm{H}+\mathrm{L}$ chains was first identified in the induced plasmacytoma line $\mathrm{T}(\mathrm{EPC}) 15$, which was shown to secrete antibodies specific to $\mathrm{PC}$. This canonical combination of $\mathrm{H}+\mathrm{L}$ chains defines the T15 idiotype (Id), which can be identified by anti-Id antibodies, either polyclonal or monoclonal $(7,8)$. Such probes have been used to show that normal mouse serum contains low levels of $\mathrm{T}^{15 \mathrm{Id}^{+}}$natural antibody with anti-PC activity but that these levels can be dramatically boosted by a primary immunization with the bacterial vaccine (9). There is strong evidence that the $\mathrm{B}$ cell precursors with this specificity develop during a critical window of development in fetal and early neonatal life (10). This developmental program can be ablated either by anti-Id-mediated deletion of T15 Id ${ }^{+}$ B cells in perinatal life (11) or by molecular intervention to prevent formation of the germline-encoded canonical T15 $\mathrm{H}+\mathrm{L}$ Ig chains (12). Both of these approaches cause a permanent loss of $\mathrm{T} 15^{+} \mathrm{B}$ cells and hence of the protective antibody. The anti-PC antibodies are among the best studied of so-called natural antibodies and represent a significant fraction of normal serum IgM found in germ-free mice (13).

\section{Natural antibodies}

\section{in atherosclerotic mice}

Shaw et al. (6) now report that this particular antibody has clearly defined specificity for modified LDL determinants. In $A p o E^{-/-}$mice, the development of atherosclerosis is paralleled by increasing titers of antibodies to OxLDL. Using 
hybridomas and a comprehensive series of antibody specificity tests, these authors show that a major portion of the response can be attributed to the expansion and activation of independently arising $\mathrm{T}_{1} 5^{+}$clones, and they confirm this finding with molecular analyses (6). Although the anti-PC response has been studied for more than 30 years, this study appears to provide the first clues to the nature of the ligand that selects for $\mathrm{T} 15^{+}$clones during development. As shown in Figure 1, other B-cell clones also respond to modified lipoprotein determinants, but the T15 response predominates in most mice. Interestingly, the anti-PC responses employ different $V_{K}$ regions but the same $J_{K} 5$ region. $J_{K} 5$ is located at the 3 -most end of the $J_{\kappa}$ locus, and its use has been interpreted as an indication that the progenitors of these $B$ cells have revised their receptors during early development to escape from self-reactivity (14). This model suggests a possible mechanism by which multispecific $\mathrm{T} 15^{+} \mathrm{B}$-cell clones may have been positively selected. These clones, which react not only with OxLDL and bacterial PC but also with apoptotic cells, may have been selected by interaction with apoptotic debris at sites of B-cell development in fetal liver and bone marrow (15), in a process that could involve receptor editing before or during the proliferation of specific B cells. Although the present authors have previously shown $(16,17)$ that deliberate immunization of hypercholesterolemic rabbits or mice expressing high levels of OxLDL lessens atherosclerosis, the role of these autoantibodies against OxLDL needs more evaluation.

The exuberant response of the $\mathrm{T} 15^{+}$ clones in $A p o E^{-/-}$mice is clearly antigen-driven, and the antigens appear to be oxidized phospholipids and/or their protein counterparts. It will be important to determine the fine specificity and the affinity of their binding to each of these species of modified LDL. Additionally, mechanisms involved in the activation of these $\mathrm{B}$ cells needs evaluation. Normally, T15 ${ }^{+}$ $\mathrm{B}$ cells can be triggered into proliferation and $\operatorname{IgM}$ antibody production in a $\mathrm{T}$-independent fashion. The role of $\mathrm{T}$ cells in the response is not yet clear, nor is it known whether switching to
IgG isotypes occurs. This possibility could be of considerable importance for understanding atherosclerotic plaque formation, since foam cells may use $\mathrm{Fc}$ receptors to take up OxLDL complexed with IgG but not IgM antibodies. Conceivably, then, chronic infection with organisms expressing PC may induce synthesis of anti-PC antibodies of IgM or even IgG isotypes, which could influence the disease process. Since the anti-PC Bcell repertoire changes during the life of the organism, the participation of natural IgM anti-PC antibodies in the disease process may vary with age (18).

One way to test these admittedly speculative ideas would be to compare the effects of $\mathrm{T}^{1} 5^{+}$antibody levels in different mouse models of atherosclerosis. In $A p o E^{-/-}$mice, the disease process begins very early, progresses steadily, and does not require high levels of dietary cholesterol. Conversely, in LDL receptor-deficient (LDLR-deficient) mice, the beginnings of atherosclerosis are not evident unless animals receive a specific diet (19). How might the production of anti-OxLDL anti-
Immature

PC-positive B cells

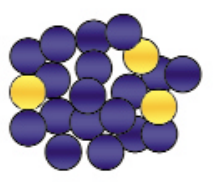

ApoE
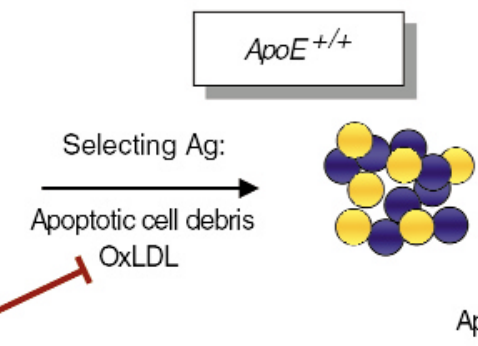

ApoE

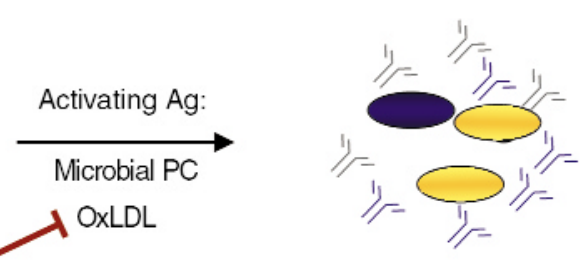

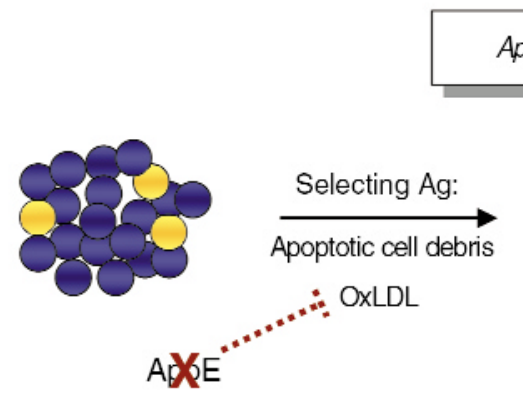

$A p o E^{-/-}$

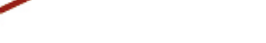

Natural Ab production

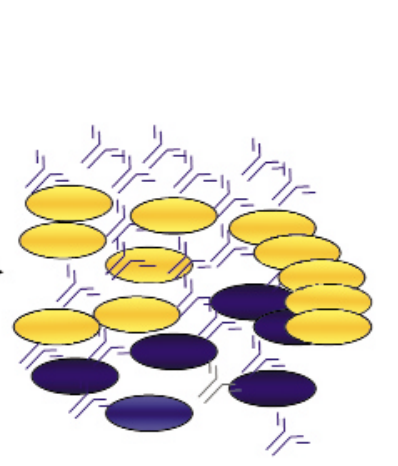

Figure 1

Potential role of OxLDL and ApoE in autoantibody production. Top: Under normal circumstances, the newly formed IgM ${ }^{+} \mathrm{B}_{\text {-cell }}$ population in the perinatal bone marrow or fetal liver can respond to millions of specific antigenic structures. Included in this population are anti-PC clones, some of which carry the T15 idiotype. In the selection step, PC epitopes on apoptotic material or OxLDL (selecting Ag) may enrich for T15/PC-specific B cells. The degree to which this occurs would be limited by the efficiency of debris-scavenging by macrophages or by the availability of ApoE. Additionally, these T15 clones may be stimulated to differentiate by the normally regulated amounts of PC or OxLDL (activating Ag). Bottom: In the absence of ApoE, accumulated OxLDL may promote a still more profound enrichment for T15 clones or may stimulate existing clones to differentiate into T15producing (natural antibody-producing) plasma cells. Either possibility would give rise to the high levels of anti-OxLDL antibodies observed in the ApoE $E^{-1-}$ mice. Circles represent B cells; ovals represent mature antibody-secreting plasma cells. Blue, $\alpha$ PC; yellow, T15/PC. 
bodies - and the natural history of the disease - be affected by providing this diet to $L D L R^{-/-}$mice only after the critical perinatal period when $\mathrm{T}^{1} 5^{+}$clones become selected? Similarly, since OxLDL has been found in fetal arteries of $A p o E^{-/-}$animals (20), this ligand may be involved in the positive selection of the emerging $\mathrm{T}_{1} 5^{+}$clones (Figure 1). The increased levels of OxLDL in the $A p o E^{-/-}$mice may amplify the normal selection process, resulting in a greatly expanded pool of these cells in the adult. The current work (6) shows that a highly conserved antibody response to PC can affect the metabolism of modified LDL, but whether it contributes to atherosclerosis, either promoting or ameliorating this condition, remains to be determined.

\section{Patterns of ligand specificity}

Shaw et al. (6) also show that $\mathrm{T}^{+} 5^{+}$antibodies bind specifically to apoptotic cells, extending their group's previous observation that antibodies to OxLDL block uptake and disposal of apoptotic cells by macrophages (21). Pattern recognition receptors such as the macrophage scavenger receptors and CD36 likewise recognize determinants on apoptotic cells. Whether $\mathrm{T}^{1} 5^{+}$antibodies interfere with these receptors or with the recently described phagocytic receptor for apoptotic cells (22) remains to be explored, but the overlapping biochemical specificity of these pattern recognition receptors and of the natural antibodies is striking in any case. In addition, type 1 macrophage scavenger receptors bind lipoteichoic acid (23), another T15 ligand.

Together, these findings provide several examples of ligands - or classes of ligands - shared by these seemingly dissimilar components of the innate immune system, and they raise intriguing questions about functional interactions between these molecules. Do T15 antibodies modulate the uptake and clearance of apoptotic cells in vivo? Does interference with this important macrophage function provide a potential source of other neoantigens and induce the proliferation of other self-reactive clones?

1. Harnett, W., and Harnett, M.M. 1999. Phosphorylcholine: friend or foe of the immune system? Immunol. Today. 20:125-129.

2. Ochsenbein, A.F., et al. 1999. Control of early viral and bacterial distribution and disease by natural antibodies. Science. 286:2156-2159.

3. Hoffmann, J.A., Kafatos, F.C., Janeway, C.A., and Ezekowitz, R.A. 1999. Phylogenetic perspectives in innate immunity. Science. 284:1313-1318.

4. Reardon, C.L., et al. 1995. Murine epidermal V gamma $5 / \mathrm{V}$ delta 1 -T-cell receptor $+\mathrm{T}$ cells respond to B-cell lines and lipopolysaccharides. J. Invest. Dermatol. 105(Suppl.):58S-61S

5. Boismenu, R., and Havran, W.L. 1997. An innate view of gamma delta T cells. Curr. Opin. Immunol. 9:57-63.

6. Shaw, P.X., et al. 2000. Natural antibodies with the T15 idiotype may act in atherosclerosis, apoptotic clearance, and protective immunity. $J$. Clin. Invest. 105:1731-1740.

7. Benca, R., Quintans, J., Kearney, J.F., Flood, P.M., and Schreiber, H. 1980. Studies on phosphorylcholine-specific $\mathrm{T}$ cell idiotypes and idiotypespecific immunity. Mol. Immunol. 17:823-831.

8. Kearney, J.F., Barletta, R., Quan, Z.S., and Quintans, J. 1981. Monoclonal vs. heterogeneous anti-H-8 antibodies in the analysis of the antiphosphorylcholine response in BALB/c mice. Eur. J. Immunol. 11:877-883.

9. McDaniel, L.S., Benjamin, W.H., Forman, C., and Briles, D.E. 1984. Blood clearance by antiphosphocholine antibodies as a mechanism of protection in experimental pneumococcal bacteremia. J. Immunol. 133:3308-3312.

10. Masmoudi, H., Mota-Santos, T., Huetz, F., Coutinho, A., and Cazenave, P.A. 1990. All T15 Id-positive antibodies (but not the majority of VHT15+ antibodies) are produced by peritoneal CD5+ B lymphocytes. Int. Immunol. 2:515-520.

11. Elliott, M., and Kearney, J.F. 1992. Idiotypic regulation of development of the B cell repertoire. Ann. NY Acad. Sci. 651:336-345.
12. Benedict, C.L., and Kearney, J.F. 1999. Increased junctional diversity in fetal $\mathrm{B}$ cells results in a loss of protective anti-phosphorylcholine antibodies in adult mice. Immunity. 10:607-617.

13. Grandien, A., et al. 1992. On the origin of natural IgM in immunoglobulin transgenic mice. Int. Immunol. 4:1153-1160.

14. Gay, D., Saunders, T., Camper, S., and Weigert, M. 1993. Receptor editing: an approach by autoreactive B cells to escape tolerance. J. Exp. Med. 177:999-1008.

15. Vakil, M., Briles, D., and Kearney, J. 1991. Antigen-independent selection of T15 idiotype during B-cell ontogeny in mice. Dev. Immunol. 1:203-212.

16. Palinski, W., Miller, E., and Witztum, J.L. 1995 Immunization of low density lipoprotein (LDL) receptor-deficient rabbits with homologous malondialdehyde-modified LDL reduces atherogenesis. Proc. Natl. Acad. Sci. USA. 92:821-825.

17. Freigang, S., Horkko, S., Miller, E., Witztum, J.L., and Palinski, W. 1998. Immunization of LDL receptor-deficient mice with homologous malondialdehyde-modified and native LDL reduces progression of atherosclerosis by mechanisms other than induction of high titers of antibodies to oxidative neoepitopes. Arterioscler. Thromb. Vasc. Biol. 18:1972-1982.

18. Nicoletti, C., Yang, X., and Cerny, J. 1993. Repertoire diversity of antibody response to bacterial antigens in aged mice. J. Immunol. 150:543-549.

19. Huszar, D., et al. 2000. Increased LDL cholesterol and atherosclerosis in LDL receptor-deficient mice with attenuated expression of scavenger receptor B1. Arterioscler. Thromb. Vasc. Biol. 20:1068-1073.

20. Napoli, C., et al. 1997. Fatty streak formation occurs in human fetal aortas and is greatly enhanced by maternal hypercholesterolemia. Intimal accumulation of low density lipoprotein and its oxidation precede monocyte recruitment into early atherosclerotic lesions. J. Clin. Invest. 100:2680-2690.

21. Chang, M.K., et al. 1999. Monoclonal antibodies against oxidized low-density lipoprotein bind to apoptotic cells and inhibit their phagocytosis by elicited macrophages: evidence that oxidationspecific epitopes mediate macrophage recognition. Proc. Natl. Acad. Sci. USA. 96:6353-6358.

22. Fadok, V.A., et al. 2000. A receptor for phosphatidylserine-specific clearance of apoptotic cells. Nature. 405:85-90.

23. Dunne, D.W., Resnick, D., Greenberg, J., Krieger, M., and Joiner, K.A. 1994. The type I macrophage scavenger receptor binds to grampositive bacteria and recognizes lipoteichoic acid. Proc. Natl. Acad. Sci. USA. 91:1863-1867. 